\title{
Uso del video como metodología de aula invertida en asignaturas de experimentación animal \\ Using the video as flipped classroom methodology in experimental animal subjects
}

\author{
María R Alvir, Pilar G Rebollar \\ mariar.alvir@upm, pilar.grebollar@upm \\ Departamento Producción Agraria \\ Escuela Técnica Superior de Ingeniería \\ Agronómica Agroalimentaria y de Biosistemas. \\ Universidad Politécnica de Madrid \\ Madrid, España
}

\begin{abstract}
Resumen- El objetivo de este estudio fue i) Evaluar el aprendizaje de los alumnos comparando las metodologías docentes, presencial y online ii) Evaluar si el alumno ha visualizado y estudiado el contenido de los videos alcanzando unos resultados de aprendizaje adecuados con la metodología de aula invertida y iii) Valorar el grado de aceptación de los materiales docentes utilizados en las prácticas por los alumnos. El nivel de conocimiento alcanzado por los alumnos con las metodologías docentes utilizadas, presencial y on line, ha sido alto y similar. Sin embargo, si se observaron diferencias en la pegunta del cuestionario donde el alumno tenía que realizar medidas y hacer cálculos, siendo más alta en presencial $(8,7)$ que online (4,3). Las calificaciones obtenidas por los alumnos fueron altas al utilizar los videos como metodología de aula invertida. Las actividades de evaluación de tipo JCloze, fué valorada positivamente por los alumnos y se puede considerar una herramienta útil para implicar y motivar al alumno en su proceso de aprendizaje. Los alumnos, a través de las encuestas realizadas, han valorado positivamente tanto las prácticas presenciales como las realizadas a través de los videos.
\end{abstract}

\section{Palabras clave: Aula invertida, Moodle, JCloze}

Abstract- The target of this study was to i) evaluate students learning by comparing classroom and online teaching methodologies. ii) Evaluate if students have visualized and studied the contents of the videos, achieving adequate learning results with the flipped classroom methodology and iii) Rate the degree of acceptance of teaching materials used in the practices by students. The level of knowledge reached by students with the teaching methodologies used, both classroom and online, has been high and similar. However, there were differences in the question where the student had to perform measurements and make calculations, being higher in faceto-face (8.7) than online (4.3). The scores obtained by the students were high when using the videos as an inverted classroom methodology. Evaluation activities of the JCloze type were positively valued by the students and can be considered a useful tool to involve and motivate the student in their learning process. The students, through the surveys, have positively valued both the face-to-face and video-based practices.

Keywords: Flipped classroom, Moodle, JCloze

\section{INTRODUCCIÓN}

La adaptación de la enseñanza universitaria hacia la implantación de los ECTS (European Credits Transfer System) ha planteado cambios en el proceso de enseñanzaaprendizaje, que afectan entre otros aspectos, a un nuevo modelo de formación centrado en el trabajo del estudiante y a que las actividades educativas diseñadas por el profesor deberán propiciar que el estudiante asuma un mayor protagonismo y responsabilidad en su aprendizaje. En este sentido, el aprendizaje invertido es un enfoque pedagógico en el que los estudiantes reciben información docente donde los recursos más frecuentes pueden ser videos apoyados en diverso material complementario de lectura que deben consultar antes de acudir al aula, en la cual, el tiempo presencial se invierte en ampliar conocimientos mediante la resolución de dudas con el/los profesor/es que les ofrecen una orientación más personalizada (Tourón y Santiago, 2013).

La incorporación de las plataformas educativas B-learning (Moodle), han servido activamente como canal de comunicación entre profesores y alumnos para implantar toda la nueva metodología docente (guías de aprendizaje, archivos PDF, links a páginas Web de interés, presentaciones PowerPoint, ficheros Excel, cuestionarios, videos, etc...). Este modelo de aprendizaje permite combinar la docencia presencial y no presencial, permitiéndonos organizar las asignaturas distribuyendo los contenidos en actividades de ambos tipos. Además, las plataformas hacen posible llevar a cabo una evaluación continua del aprendizaje de los alumnos, mediante la realización de los ejercicios de autoevaluación y representan una herramienta eficaz para adecuar el ritmo de enseñanza al ritmo de aprendizaje del alumno (Portaencasa et al, 2006).

Desde el curso 2003-2004 se están utilizando las plataformas B-learning (Aula web y Moodle) en algunas asignaturas, impartidas por el Grupo de Innovación ZOOINNOVA de la Escuela Técnica Superior de Ingeniería 
Agronómica, Agroalimentaria y de Biosistemas de la Universidad Politécnica de Madrid, fundamentalmente para que accedieran a la información de la asignatura y realizar ejercicios de autoevaluación (Villamide et al., 2006). En el curso 2005-2006, se utilizaron para la realización de exámenes parciales y con el objetivo de no perder el tiempo copiando apuntes se les colgaron las presentaciones de las clases como apoyo a la clase presencial (Nicodemus et al., 2006). Además, se propusieron metodologías más activas (trabajos en grupo vs apuntes de clase) para lograr una motivación en el alumno y que se implicara en el proceso de aprendizaje (Alvir et al., 2007) así como hacer un seguimiento del tiempo dedicado al estudio de diferentes temas del programa de la asignatura de Zootecnia, a los ejercicios de autoevaluación en la plataforma Moodle y al tiempo dedicado a la realización y presentación de trabajo de grupo con el fin de ir adaptando los contenidos y el proceso de enseñanza-aprendizaje a los créditos ECTS (Alvir et al, 2009).

\section{CONTEXTO}

El empleo de animales de experimentación para la docencia es un hecho en el área de Producción Animal en la que es necesario sentar las bases anatómicas de determinados sistemas orgánicos (digestivo y reproductor) para entender su funcionamiento, y poder aplicar los conocimientos adquiridos en una explotación ganadera. Según el RD 53/2013 "la enseñanza superior o la formación para la adquisición o mejora de las aptitudes profesionales" es uno de los fines en el que se puede utilizar animales. Según este RD se debe fomentar el uso de métodos alternativos a la experimentación con animales vivos, donde el número de animales utilizados en los procedimientos debe reducirse al mínimo. En este sentido, en las prácticas de la asignatura de "Fisiología Animal" donde se utilizan animales de experimentación, se pretende reducir el número de animales sin detrimento de los conocimientos que se deben de adquirir. Para ello se va a emplear la metodología del aula invertida donde los estudiantes reciben información virtual a través de los videos, protocolos o guías de disección, pueden planificar y gestionar mejor su tiempo en función de sus necesidades y aunque el estudiante aprende solo, este aprendizaje estará dirigido por el profesor.

El objetivo de este estudio fue i) Evaluar el aprendizaje de los alumnos comparando las metodologías docentes, presencial y online ii) Evaluar si el alumno ha visualizado y estudiado el contenido de los videos alcanzando unos resultados de aprendizaje adecuados con la metodología de aula invertida y iii) Valorar el grado de aceptación de los materiales docentes utilizados en las prácticas por los alumnos.

\section{DESCRIPCIÓN}

Fisiología animal es una asignatura obligatoria de 4 créditos ECTS cursada, en $2^{\circ}$ curso en el $2^{\circ}$ semestre, por todos los alumnos que opten al título de Ingeniería y Ciencia Agronómica. La asignatura está estructurada en seis bloques de unidades temáticas a impartir en 2 grupos. Cada bloque se evalúa de forma independiente en base a los resultados del examen parcial y al trabajo continuo realizado, según la siguiente ecuación: $0,60 \times$ examen parcial $+0,15 \times$ prácticas + $0,20 \times$ trabajo en grupo $+0,05 \times$ ejercicios de autoevaluación.
Los alumnos que superasen la asignatura a través de la evaluación continua no tenían que presentarse al examen final.

En este trabajo nos vamos a centrar en los resultados obtenidos, por los alumnos matriculados en la asignatura de Fisiología Animal en el curso 2016-2017, en dos prácticas presenciales una correspondiente a la disección del sistema digestivo del conejo y la otra a la disección del sistema reproductor de la coneja y del conejo. En estas prácticas los alumnos podían elegir hacerlas presencial en el laboratorio u online. Por otro lado, se realizaron además para todos los alumnos dos prácticas online, mediante la visualización de videos, correspondientes a la técnica de inseminación artificial del conejo y a la disección de la gallina. Todas estas prácticas corresponden a tres bloques de las unidades temáticas del programa.

En la plataforma Moodle, en la semana correspondiente a la programación de las prácticas presenciales y online todos los alumnos disponían de los protocolos donde se detallaban como tenían que realizar las disecciones junto con fotografías de los órganos que tenían que visualizar además de los correspondientes vídeos. Los estudiantes deberían elegir en una encuesta personal colgada en Moodle si deseaban realizarlas de manera presencial (en el laboratorio con animales) o no. Se prepararon las prácticas de acuerdo al número de alumnos que habían decidido hacerla presencial con los animales necesarios.

Cada práctica presencial duraba 1,5 horas. Los profesores estaban apoyando y confirmando que el trabajo del alumno era autónomo (lo hacían en parejas). Se hacían preguntas sobre el procedimiento que estaban realizando en cada una de las prácticas, insistiendo sobre todo si habían comprendido el proceso de la disección y la identificación de los diferentes órganos.

En la práctica del sistema digestivo tenían que realizar determinaciones y medidas en el animal directamente (longitudes, pesos absolutos, relativos...) Posteriormente, con la documentación disponible y con los datos que habían tomado, tenían que comprobar, estudiar y discutir con los restantes compañeros si los datos eran correctos y crear entre todos un documento que estaría disponible en Moodle para todos los estudiantes, tanto los que hicieron la práctica presencial o los que vieron solo el vídeo.

Al finalizar cada práctica y en ese mismo día se les activaba un cuestionario general de autoevaluación en la plataforma Moodle de 10 preguntas, a responder en 15 minutos y solo lo podían realizar una vez. Una de las preguntas correspondiente al cuestionario de la disección del sistema digestivo era referente al cálculo de los pesos relativos de diferentes órganos.

Para las prácticas diseñadas únicamente online se realizaron dos videos que estuvieron disponibles en la plataforma Moodle durante una semana y se cerraban en el momento que se activaba un cuestionario general. Además, y antes de realizar este cuestionario, con el objetivo de implicar y motivar al estudiante en su proceso de aprendizaje, creamos a través de Moodle y con el programa Hotpotatoes actividades de evaluación de tipo JCloze, que permite a partir de un texto 
generar huecos que el alumno debe rellenar, y que nos sirviera para evaluar si el alumno había visualizado y estudiado el contenido del video alcanzando los resultados de aprendizaje necesarios para aprobar la asignatura.

Para averiguar la opinión de los alumnos sobre las metodologías docentes utilizadas en las prácticas, se les pasó el día del último examen de evaluación continua una encuesta donde debían contestar a diferentes preguntas valorándolas de 1 a 5 (1 nada de acuerdo y 5 totalmente de acuerdo).

\section{Resultados}

A. Evaluación del aprendizaje de los alumnos comparando metodologías docentes (presencial - online)

De los 54 alumnos matriculados 46 respondieron que la práctica del sistema digestivo la hacían en la modalidad presencial, 5 respondieron que online y 3 alumnos que no contestaron a la opción (presencial y online). Únicamente contestaron al cuestionario 49 alumnos (4 alumnos que la hicieron presencial no contestaron al cuestionario y 1 alumno que no contesto a la elección de la práctica tampoco lo hizo).

En la figura 1 se indican las calificaciones de la práctica del sistema digestivo modalidad presencial y online correspondientes al cuestionario general y a una pregunta (de opción calculada) del cuestionario. Puede observarse que no existieron diferencias en las calificaciones del cuestionario general obtenidas por los alumnos que realizaron la práctica presencial y online (media 7,75). Sin embargo, sí se observan diferencias en aquella pregunta del cuestionario donde el alumno tenía que realizar medidas y hacer cálculos, donde la calificación que obtienen los alumnos que lo hicieron presencial fue mucho más alta $(8,7)$ que la que la hicieron online $(4,3)$, si bien solo fueron 7 alumnos los que eligieron esta última opción y disponían de un documento colgado en Moodle con los resultados obtenidos por sus compañeros que optaron por la práctica presencial.

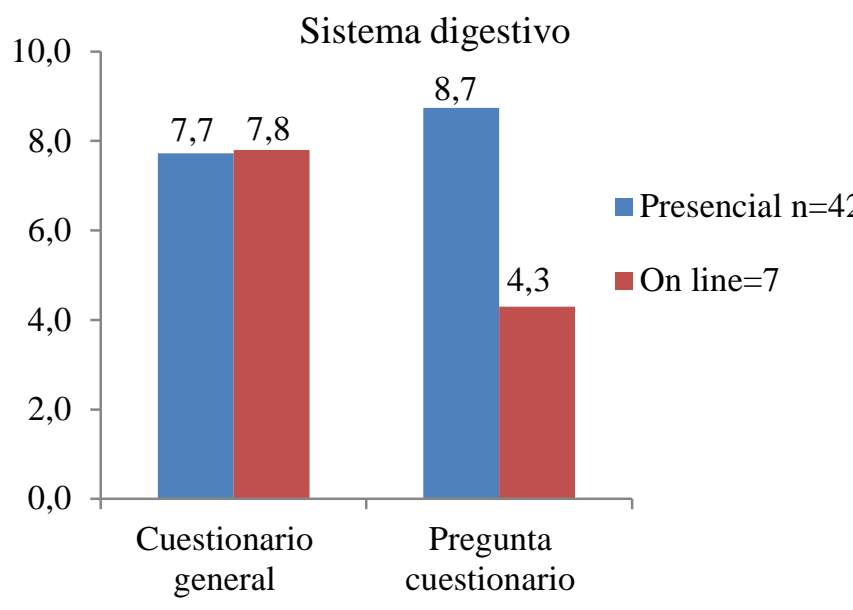

Figura 1. Calificaciones de la práctica de disección del sistema digestivo presencial y online: Cuestionario general y pregunta cuestionario.
En la segunda práctica correspondiente al sistema reproductor, únicamente eligieron la opción presencial 28 alumnos y 19 alumnos eligieron online (figura 2). Se observa, que no existieron diferencias en las calificaciones de los cuestionarios realizados en la modalidad presencial y on line, tanto en el cuestionario para el macho (9,5 presencial vs 9,2 on line) como en el cuestionario para la hembra (7,9 presencial vs 7,2 on line). Esta menor calificación alcanzada en la práctica con la hembra, se debería más a la complejidad de la diseccción de la hembra que a la metodologia docente empleada. Con estos resultados, se podría deducir que el nivel de conocimientos comparando ambas metodologias es bastante similar, por lo que en estas asignaturas en las que se utilizan animales de experimentación, nos permitiría reducir el número de animales vivos, si bien sería conveniente seguir investigando en otras asignaturas experimentales con actividades prácticas de este tipo.

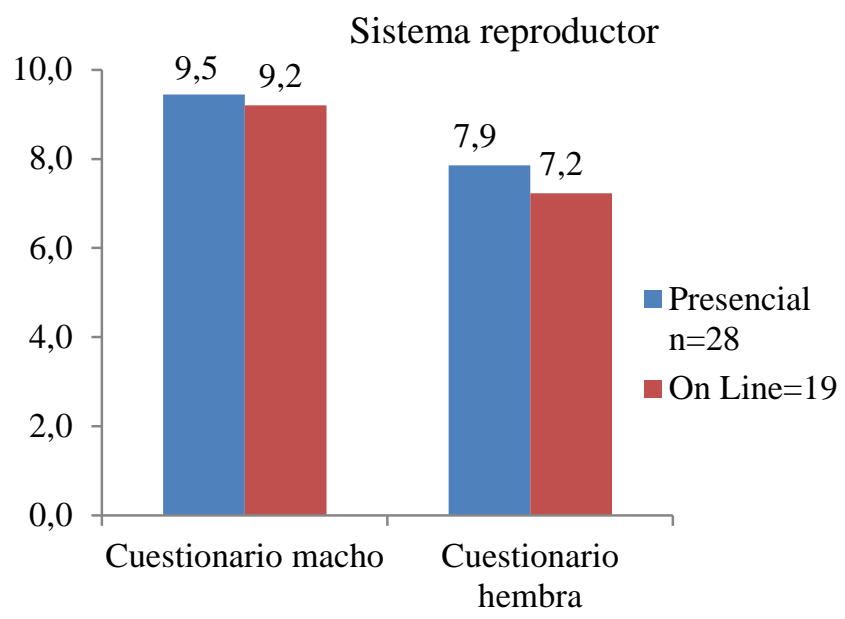

Figura 2. Calificaciones de la práctica de disección del sistema reproductor presencial y on line: Cuestionario macho y cuestionario hembra

\section{B. Evaluación de los resultados de aprendizaje utilizando únicamente videos como metodología de aula invertida. .}

El número de alumnos que realizaron los cuestionarios fue diferente en cada una de las prácticas. Para la práctica de la inseminación artificial, realizaron el cuestionario JCloze 34 alumnos y el cuestionario general 40 y para la disección de la gallina, el número de alumnos que hizo el cuestionario JCloze fue más bajo $(n=26)$ y el cuestionario general 44 alumnos.

En la figura 3 se indican los resultados correspondientes a estos cuestionarios. Puede observarse que el aprendizaje alcanzado de las prácticas, tanto de la inseminación artificial como la disección de la gallina a través de los videos, muestra calificaciones altas. En la práctica de inseminación artificial, el hacer antes la actividad JCloze incidió en una superior nota en el cuestionario general (7,6 vs 8,1), pero para la práctica de disección de la gallina las notas fueron altas y similares entre el JCloze y el cuestionario general respectivamente (7,5 vs 7,3); si bien hay que indicar que los alumnos mostraron menos interés en realizar la actividad JClose ya que solo lo realizaron 
26 alumnos, quizá atribuible a que estaban a final de curso, y el tiempo disponible a realizar las actividades fuera menor.

\section{Valoración de los alumnos sobre el grado de aceptación de los materiales docentes utilizados en las prácticas}

Para averiguar la opinión de los alumnos sobre los videos se les pasó, el último día de clase cuando realizaron el último examen de evaluación continua, una encuesta donde debían contestar a diferentes preguntas valorándolas de 1 a 5 (1 nada de acuerdo y 5 totalmente de acuerdo). Los alumnos han valorado positivamente el grado de aprendizaje logrado con las prácticas presenciales $(4,8)$ y con las prácticas online $(4,5)$. Para ellos, la utilización de los videos para presentar parte de los contenidos de la asignatura ha sido un recurso útil para su aprendizaje $(4,6)$. Por otro lado, una vez vistos los vídeos, a los alumnos les ha sido fácil contestar a las preguntas de los cuestionarios tipo JCloze $(4,2)$ y al cuestionario en general $(4,3)$.

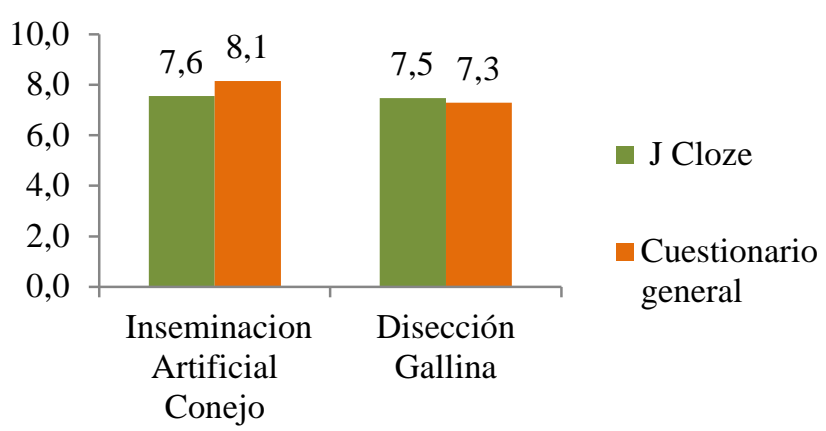

Figura 3. Calificaciones de las prácticas de inseminación artificial del conejo y disección de la gallina utilizando actividades de evaluación tipo JCloze y cuestionario general.

En la figura 4 se exponen las puntuaciones de los alumnos a la siguiente pregunta:" He realizado los ejercicios de JCloze relacionados con la visualización de los videos para...." Se observa que han valorado muy positivamente esta actividad tanto para obtener más nota en la asignatura $(4,5)$, implicarse más en el aprendizaje $(4,4)$ y para mejorar la compresión del contenido del contenido del video $(4,2)$ vídeo.

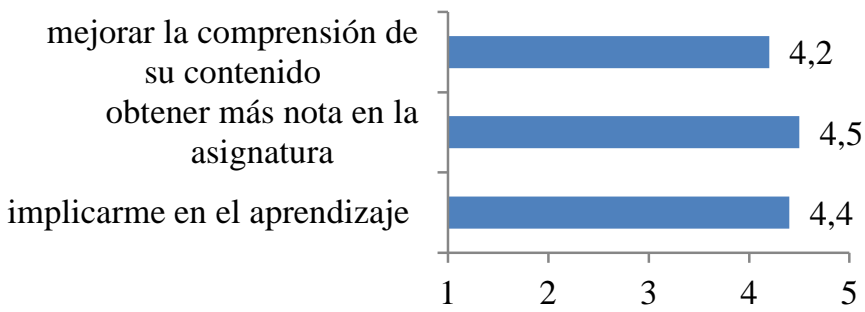

Figura 4. Puntuaciones de los alumnos sobre: "He realizado los ejercicios tipo JCloze relacionados con la visualización de los videos para...”.
El nivel de conocimiento alcanzado por los alumnos con las metodologías docentes utilizadas, presencial y on line, ha sido alto y similar.

Las calificaciones obtenidas por los alumnos fueron altas al utilizar los videos como metodología de aula invertida.

Los alumnos han valorado positivamente tanto las prácticas presenciales como las realizadas a través de los videos.

Las actividades de evaluación de tipo JCloze, fueron valoradas positivamente por los alumnos y se puede considerar una herramienta útil para implicar y motivar al alumno en su proceso de aprendizaje.

\section{AGRADECIMIENTOS}

Los autores desean agradecer a la Universidad Politécnica de Madrid la financiación recibida por el Proyecto de Innovación Educativa. A todos los alumnos de la asignatura Fisiología Animal (Curso 2016-17) que han participado en las encuestas y en especial a la alumna María Rodríguez Francisco como apoyo en las prácticas presenciales y a María Sánchez-Bayo como becaria del citado proyecto.

\section{REFERENCIAS}

Alvir, M. R., Nicodemus, N., Alegre, Y., Saiz, A., Solis, I., Garcia, J. Gonzalez, J. (2007). Experiencia docente como factor motivador del alumno. Actas de las II Jornadas de Innovación educativa, Zamora. Pp. 102-105.

Alvir, M. R., Nicodemus, N., Garcia, J., Menoyo, D., Gonzalez, J., Hernandez, I., Rubio, J.M. (2009) Valoración de actividades propuestas en Zootecnia I: hacia la adaptación al ECTS. V Congreso Iberoamericano de docencia universitaria: Enseñar y aprender en la universidad del siglo XXI: propuestas y condiciones, Valencia. pp. 471-473. ISBN 978-84-8363413-4

Nicodemus, N., Alvir, M. R., Garcia, J. Gonzalez, J., Menoyo, D., Alegre, Y., Solis, I. (2006). Evaluación contínua mediante el uso de las plataformas B-Learning Moodle y Aulaweb en la asignatura troncal Zootecnia I. Actas de las II Jornadas de Innovación Educativa, Zamora. pp. 7984.

Portaencasa, R., Perez, A.B., Garcia, J., Fernandez, L.E. (2006). Moodle orientado a la enseñanza en modalidad mixta o B-learning. Instituto de Ciencias de la Educación (ICE). Madrid: Universidad Politécnica de Madrid.

Tourón, J., Santiago, R. (2013). El modelo flipped learning y el desarrollo del talento en la escuela. Revista de Educación. pp. 196-231.

Villamide, M. J., Alvir, M. R., Alegre, Y., Garcia, J., Nicodemus, N. (2006). El uso del sistema b-learning mediante la plataforma Aula Web en la asignatura troncal Zootecnia I en la E.T.S.I. Agrónomos de Madrid. Actas de las I Jornadas de Innovación Educativa, Zamora. pp. 480-487.

\section{CONCLUSIONES}

